\title{
Duality and Absence of Locally Generated Superselection Sectors for CCR-Type Algebras
}

\author{
W. Driessler ${ }^{\star 1}$ \\ Department of Mathematics, Bedford College, Regent's Park, London, UK
}

\begin{abstract}
We isolate an abstract algebraic property which implies duality in all locally normal, irreducible representations of a quasilocal $C^{*}$-algebra if it holds together with two more specific conditions. All these conditions holding for the CCR-algebra in $d \geqq 2$ space time dimensions duality follows for representations of the two-dimensional CCR-algebra generated by pure Wightman states of $P(\Phi)_{2}$-theories. We then show that algebras of this kind have no nontrivial locally generated superselection sectors which for $d \geqq 3$ yields a first approximation to a quantum analogue of Derrick's theorem.
\end{abstract}

\section{Preliminaries}

Viewing the central role which duality plays in the abstract theory of superselection sectors [1] it is unfortunate that so far it has not been possible to prove this property in any nontrivial case (see, however, [2] where a suitable $C^{*}$-algebra is constructed from the given one - this will, however, hardly be representation independent). On account of this the present paper is good news for it remedies this situation. On the other hand the very property which makes this possible also implies that sectors generated by local automorphisms from a vacuum sector are unitarily equivalent to it - therefore in these cases nontrivial superselection sectors in the sense of [1] do not exist. Thus the present paper raises as many questions as it solves.

We let $\mathfrak{A}$ be our quasilocal $C^{*}$-algebra with its net of local von Neumann algebras $\{\mathscr{R}(\mathcal{O})\}$. To simplify matters we shall assume $\mathscr{R}(\mathcal{O})$ to be a factor if $\mathcal{O}$ is a bounded double cone (b.d.c.). For any b.d.c. $\mathcal{O}$ we denote by $\mathcal{O}^{x}$ the open interior of its causal complement and by $\mathfrak{A}\left(\mathscr{O}^{x}\right)$ the $C^{*}$-subalgebra of $\mathfrak{A}$ generated by all $\mathscr{R}(\widetilde{\mathcal{O}})$ where $\tilde{\mathcal{O}}$ runs through all b.d.c.'s with $\tilde{\mathcal{O}} \ll \mathcal{O}^{x}$, i.e. $\overline{\tilde{O}} \subset \mathcal{O}^{x}$. This definition is a matter of convenience and must be modified if there are observables in $\mathcal{O}^{x}$ which cannot be approximated by observables localized in b.d.c.'s contained in

* Supported by Science Research Council

1 Present Address: University of Osnabrück, Postfach 4469, D-4500 Osnabrück 
$\mathcal{O}^{x}$ as in the case of the electromagnetic field. By $\mathscr{R}(\mathcal{O})^{c}$ we denote the relative commutant of $\mathscr{R}(\mathcal{O})$ in $\mathfrak{A}$. Thus for fixed $\mathcal{O} \mathfrak{U}\left(\mathcal{O}^{x}\right)$ and $\mathscr{R}(\mathcal{O})^{c}$ are representation independent objects and $\mathfrak{U}\left(\mathcal{O}^{x}\right) \subset \mathscr{R}(\mathcal{O})^{c}$ - the inclusion being proper in general as shown by the example of the CCR-algebra.

We shall have occasion to take double commutants of subalgebras of $\mathfrak{A}$ in various representations. Care will be taken to indicate which one is meant in each instance. In the following we shall only consider b.d.c.'s subtended by an interval $(-a, a)$ on the time axis. This simplifies the geometry and in concrete cases the covariance properties which do not enter the subsequent arguments will take care of the other ones.

We now define the crucial property " $F$ " where " $F$ " stands for "funnel" since property $F$ allows to construct special funnels for $\mathfrak{U}[3]$.

Definition. We say that $\mathfrak{A}$ has property $F$ if for all pairs of b.d.c.'s $\mathcal{O}_{1}, \mathcal{O}_{2}$ with $\mathcal{O}_{1} \ll \mathcal{O}_{2}$ there exists a type $I_{\infty}$ factor $\mathfrak{M} \subset \mathfrak{U}$ such that $\mathscr{R}\left(\mathcal{O}_{1}\right) \subset \mathfrak{M} \subset \mathscr{R}\left(\mathcal{O}_{2}\right) .^{2}$

We claim that the CCR-algebra in $d \geqq 2$ space-time dimensions has this property. In this case one already knows that duality holds in the Fock-representation (for the nonspecialist we shall briefly discuss the case $d=2$ below) thus it is only necessary to prove existence of a normal product state for the von Neumann algebra $\mathscr{R}\left(\mathscr{R}\left(\mathcal{O}_{1}\right), \mathscr{R}\left(\mathcal{O}_{2}^{x}\right)\right)$ where $\mathscr{R}\left(\mathcal{O}_{2}^{x}\right)=\pi_{\mathscr{F}}\left(\mathfrak{A}\left(\mathcal{O}^{x}\right)\right)^{\prime \prime}$ (see the discussion in [4] where the latter has been proven for $d=4$ ). Though this proof can be generalized to cover all dimensions $d \geqq 2$ it is much easier to deduce it from the implementability of local internal $S O(2)$ field rotations in the tensor product of the Fock representation with itself [5] for $d=2$. The latter method in turn extends to higher dimensions if one uses essential selfadjointness of the corresponding current operator when smeared with testfunctions of the form $g(x) \chi(t)$ with $g \in \mathscr{D}\left(\mathbb{R}^{d-1}\right)$ and $\chi$ the characteristic function of an interval. Since we are mainly interested in application to $P(\phi)_{2}$-models we shall not go into details.

\section{Duality}

After these preparations we can state the following theorem:

Theorem 1. Let $\mathfrak{U}$ have property $F$ and assume further:

(1) There exists an irreducible, locally normal representation (i.l.n.r.) $\pi_{0}$ of $\mathfrak{A}$ in which duality holds for all b.d.c.'s in the sense that $\pi_{0}\left(\mathfrak{H}\left(\mathcal{O}^{x}\right)\right)^{\prime \prime}=\mathscr{R}(\mathcal{O})_{\pi_{0}}^{\prime}$.

(2) In $\pi_{0}$ there exists for any pair of b.d.c.'s $\mathcal{O}_{1} \mathcal{O}_{1}$ with $\mathcal{O} \ll \mathcal{O}_{1}$ another b.d.c. $\mathcal{O}_{2}$ with $\mathcal{O}_{1} \ll \mathcal{O}_{2}$ such that $\mathscr{R}(\mathcal{O})_{\pi_{0}}^{\prime}$ is generated by $\pi_{0}\left(\mathfrak{U}\left(\mathcal{O}_{1}^{x}\right)\right)^{\prime \prime}=\mathscr{R}\left(\mathcal{O}_{1}\right)_{\pi_{0}}^{\prime}$ and $\left[\mathfrak{U}\left(\mathcal{O}^{x}\right) \cap\right.$ $\left.\mathscr{R}\left(\mathrm{O}_{2}\right)\right]_{\pi_{0}}^{\prime \prime}$.

(3) For any triple of b.d.c.'s $\mathcal{O}_{,} \mathcal{O}_{1}, \mathcal{O}_{2}$ with $\mathcal{O} \ll \mathcal{O}_{1} \ll \mathcal{O}_{2}$ there exists a further b.d.c. $\mathcal{O}_{3}$ with $\mathcal{O}_{2} \ll \mathcal{O}_{3}$ and such that $\pi_{0}\left(\mathscr{R}\left(\mathcal{O}_{1}\right)^{c} \cap \mathscr{R}\left(\mathcal{O}_{2}\right)\right) \subset\left[\mathfrak{U}\left(\mathcal{O}^{x}\right) \cap \mathscr{R}\left(\mathcal{O}_{3}\right)\right]_{\pi_{0}}^{\prime \prime}$.

Then duality holds for all b.d.c.'s in every i.l.n.r. of $\mathfrak{A}$.

2 We shall assume the $\mathscr{R}(\mathcal{O})$ to be countably decomposable; it then easily follows that the factors $\mathfrak{M}$ also are countably decomposable. 
Remark. Assumption 2 is a regularity condition which should hold in all sufficiently well behaved theories, in particular if local algebras are generated from spectral projections of field operators in a Wightman theory of scalar fields obeying the assumptions of [6]. Assumption 3 is physically significant since it excludes certain observables (standard example: magnetic flux) from the theory. Since from the results of [1] we would expect duality to hold in abelian sectors if it holds in the vacuum sector the above assumptions are probably stronger than necessary. However that may be, for the CCR-algebra in $d \geqq 2$ we have the far stronger property that for all b.d.c.'s $\mathcal{O}, \mathcal{O}_{1}$ with $\mathcal{O} \ll \mathcal{O}_{1}$ in the Fock representation we have: $\mathscr{R}(\mathcal{O})_{\pi \mathscr{F}}^{\prime} \cap \mathscr{R}\left(\mathcal{O}_{1}\right)=\mathscr{R}\left(\mathcal{O}^{x} \cap \mathcal{O}_{1}\right)$. For $d=4$ this has first been shown in [7]. It remains true for $d=2$, however, since a) antilocality for the operators $\Omega^{\alpha}=\left(m^{2}+k^{2}\right)^{\alpha}, \alpha \in \mathbb{R} \backslash \mathbb{Z}$, is dimension independent [8];b) it is easy to show directly that for $\mathcal{Q}$ either an interval of the disjoint unions of two interval in $(t=0)$ one

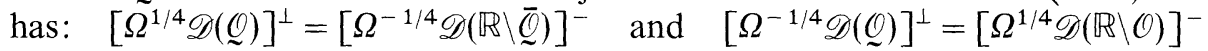
in $L^{2}(\mathbb{R})$; therefore the subspaces relevant for application of e.g. [9] are in generic position which is what is needed to get the assertion. For the CCR-algebra, however, it is clear that $\mathscr{R}\left(\mathcal{O}^{x} \cap \mathcal{O}_{1}\right)$ is equal to $\pi_{\mathfrak{F}}\left[\mathfrak{U}\left(\mathcal{O}^{x}\right) \cap \mathscr{R}\left(\mathcal{O}_{1}\right)\right]^{\prime \prime}$ there all the conditions of the theorem are fulfilled in this case.

From the commutant theorem for tensor products it follows that this is equally true for the quasilocal system resulting from the $U(1)$ localization of the complex scalar field [10]. This example will be discussed in more detail in the next section.

The proof of the theorem is broken down in a number of easy steps. The methods used are (surprisingly) elementary except possibly for the notion of conditional expectation in von Neumann algebras (C.E.'s). For the convenience of the reader we list those properties of C.E.'s which we shall need (for more details see e.g. the appendix of [11]):

Let $\mathfrak{M} \subset \mathscr{L}(\mathscr{H})$ be a von Neumann algebra and $P: \mathscr{L}(\mathscr{H}) \rightarrow \mathfrak{M}$ a C.E. $P$ is linear and satisfies:

(a) $\|P(A)\| \leqq\|A\|$ for all $A \in \mathscr{L}(\mathscr{H})$.

(b) $P(A B)=P(A) B$ and $P(B A)=B P(A)$ for all $A \in \mathscr{L}(\mathscr{H}), B \in \mathfrak{M}$; in particular $P([A, B])=[P(A), B]$ for such pairs.

The von Neumann algebras which are the ranges of C.E.'s are called injective and all type I factors are injective [12].

We fix the b.d.c. $\mathcal{O}$ for which we want to prove duality and choose a sequence $\left\{\mathcal{O}_{n}\right\}$ of b.d.c.'s such that $\bigcap_{n} \mathcal{O}_{n}=\overline{\mathcal{O}}$ and $\mathcal{O}_{n+1} \ll \mathcal{O}_{n}$ for all $n$. Property $\mathrm{F}$ then gives us a sequence of type I factors $\left\{\mathfrak{M}_{n}\right\}$ such that $\mathscr{R}\left(\mathcal{O}_{n}\right) \supset \mathfrak{M}_{n} \supset \mathscr{R}\left(\sigma_{n+1}\right)$. Therefore the sequence $\mathfrak{M}_{n}$ is decreasing and from duality in $\pi_{0}$ we see that $\bigcap \mathfrak{M}_{n}=\mathscr{R}(\mathcal{O})$.

Next we choose another sequence of b.d.c.'s $\left\{\widetilde{\mathcal{O}}_{k}\right\} \uparrow \mathbb{R}^{d}{ }^{n}$ with $\widetilde{\mathcal{O}}_{k} \ll \widetilde{\mathcal{O}}_{k+1}$ and $\tilde{\mathcal{O}}_{k} \gg \mathcal{O}_{n}$ for all $k, n$. This yields a sequence $\left\{\mathfrak{N}_{k}\right\}$ of type I factors such that $\mathscr{R}\left(\widetilde{\mathscr{O}}_{k}\right) \subset$ $\mathfrak{N}_{k} \subset \mathscr{R}\left(\widetilde{\mathcal{O}}_{k+1}\right)$. Thus $\mathfrak{A}$ is the norm closure of $\bigcup_{k} \mathfrak{N}_{k}$ and a representation of $\mathfrak{A}$ is locally normal if it is locally normal with respect to the type I funnel $\left\{\mathfrak{N}_{k}\right\}$. Note that by construction $\mathfrak{N}_{k} \supsetneqq \mathfrak{M}_{n}$ for all $n, k$. 
Lemma 1. Let $\pi$ be any i.l.n.r. of $\mathfrak{A}$. Then for each of the $\mathfrak{M}_{n}$ we have $\left[\pi\left(\mathfrak{M}_{n}^{c}\right)\right]^{\prime \prime}=$ $\left(\mathfrak{M}_{n}\right)_{\pi}^{\prime}$.

Proof. Consider the von Neumann algebra $\mathscr{R}\left(\left[\pi\left(\mathfrak{M}_{n}^{c}\right)\right]^{\prime \prime}, \mathfrak{M}_{n}\right)$. It contains all the factors $\mathfrak{N}_{k}$ and so is equal to $\mathscr{L}\left(\mathscr{H}_{\pi}\right)$. Since $\mathfrak{M}_{n}$ is type I and all type I factors are "coupled" we have $\left[\pi\left(\mathfrak{M}_{n}^{c}\right)\right]^{\prime \prime}=\left(\mathfrak{M}_{n}\right)_{\pi}^{\prime}$.

Remark. The same argument shows that $\mathscr{R}(\mathcal{O})$ and $\left[\pi\left(\mathscr{R}(\mathcal{O})^{c}\right)\right]^{\prime \prime}$ generate $\mathscr{L}\left(\mathscr{H}_{\pi}\right)$ but since $\mathscr{R}(\mathcal{O})$ is in general not type I we cannot conclude from this that $\left[\pi\left(\mathscr{R}(\mathcal{O})^{c}\right)\right]^{\prime \prime}=\mathscr{R}(\mathcal{O})_{\pi}^{\prime}$. Using lemma 1 we get, however:

Lemma 2. In every i.l.n.r. $\pi$ of $\mathfrak{U}$ we have $\left[\pi\left(\mathscr{R}(\mathcal{O})^{c}\right)\right]^{\prime \prime}=\mathscr{R}(\mathcal{O})_{\pi}^{\prime}$.

Proof. We have: $\left[\bigcup_{n} \pi\left(\mathfrak{M}_{n}^{c}\right)\right]^{\prime \prime}=\left[\bigcup_{n}\left(\mathfrak{M}_{n}\right)_{\pi}^{\prime}\right]^{\prime \prime}=\left(\bigcap_{n} \mathfrak{M}_{n}\right)_{\pi}^{\prime}=\mathscr{R}(\mathcal{O})_{\pi}^{\prime}$.

Since clearly $\mathscr{R}^{n}(\mathcal{O})^{c} \supset \bigcup_{n} \mathfrak{M}_{n}^{c}$ we are done.

The crucial step is contained in

Lemma 3. We have $\mathscr{R}(\mathcal{O})^{\mathfrak{c}}=\left(\bigcup_{k}\left(\mathscr{R}(\mathcal{O})^{\mathfrak{c}} \cap \mathfrak{N}_{k}\right)\right)^{- \text {norm }}$.

Proof. Let $A \in \mathscr{R}(\mathcal{O})^{c}$ arbitrary and choose $\varepsilon>0$. Then there is a $k \in \mathbb{N}$ and a $A_{\varepsilon} \in \mathfrak{N}_{k}$ such that $\left\|A-A_{\varepsilon}\right\| \leqq \varepsilon$. As discussed previously, there exists a C.E. $P: \mathscr{L}\left(\mathscr{H}_{\pi_{0}}\right) \rightarrow$ $\mathfrak{N}_{k}$. By property a) of C.E.'s we have: $\left\|P(A)-A_{\varepsilon}\right\|=\left\|P\left(A-A_{\varepsilon}\right)\right\| \leq\left\|A-A_{\varepsilon}\right\| \leq \varepsilon$. For $B \in \mathscr{R}(\mathcal{O}) \subset \mathfrak{N}_{k}$ and $A \in \mathscr{R}(\mathcal{O})^{c}$ we have by b):

$$
\begin{aligned}
& {[P(A), B]=P([A, B])=0 ; \text { therefore } P(A) \in \mathscr{R}(\mathcal{O})^{c} \cap \mathfrak{N}_{k} \text { and }} \\
& \|P(A)-A\| \leq 2 \varepsilon, \quad \text { q.e.d. }
\end{aligned}
$$

Proof of Theorem. Duality in representation $\pi_{0}$ implies that every element in $\pi_{0}\left(\mathscr{R}(\mathcal{O})^{c}\right)$ is approximated strongly by elements in $\pi_{0}\left(\mathfrak{A}\left(\mathcal{O}^{x}\right)\right)$. The previous lemmas show then that duality will hold in every i.l.n.r. if for a given factor $\mathfrak{H}_{k}$ we can find a b.d.c. $\mathcal{O}_{\mathfrak{M}_{k}}$ such that:

$$
\left[\mathfrak{U}\left(\mathcal{O}^{x}\right) \cap \mathscr{R}\left(\mathcal{O}_{\mathfrak{N}_{k}}\right)\right]_{\pi_{0}}^{\prime \prime} \cap \mathfrak{N}_{k}=\mathscr{R}(\mathcal{O})^{c} \cap \mathfrak{N}_{k}
$$

Namely, $\mathfrak{A}\left(\mathcal{O}^{x}\right) \cap \mathscr{R}\left(\mathcal{O}_{\mathfrak{N}_{k}}\right)$ is a $C^{*}$-subalgebra of some large local type I factor $\mathfrak{N}_{l}$.

Then, since $\mathfrak{U}$ is simple [13], all its representations are isomorphisms so that the map $\pi_{0}(A) \mapsto \pi(A)$ is a normal isomorphism for each $\mathfrak{N}_{l}$ and thus we can replace $\pi_{0}$ with an arbitrary i.l.n.r. $\pi$ of $\mathfrak{U}$ on the left hand side of the above formula.

Now by the first part of Assumption 2 we can find b.d.c.'s $\mathcal{O}_{1}, \mathcal{O}_{2}$ with $\mathcal{O} \ll$ $\mathcal{O}_{1} \ll \mathcal{O}_{2}$ and $\mathscr{R}\left(\mathcal{O}_{2}\right) \subset \mathfrak{N}_{k}$ for some $k \in \mathbb{N}$ such that $\mathscr{R}\left(\mathfrak{A}\left(\mathcal{O}_{1}^{x}\right)_{\pi_{0}}^{\prime \prime},\left[\mathfrak{A}\left(\mathcal{O}^{x}\right) \cap \mathscr{R}\left(\mathcal{O}_{2}\right)\right]_{\pi_{0}}^{\prime \prime}\right)=$ $\mathscr{R}(\mathcal{O})_{\pi_{0}}^{\prime}$ which, taking commutants, gives $\mathscr{R}\left(\mathcal{O}_{1}\right) \cap\left[\mathfrak{U}\left(\mathcal{O}^{x}\right) \cap \mathscr{R}\left(\mathcal{O}_{2}\right)\right]_{\pi_{0}}^{\prime}=\mathscr{R}(\mathcal{O})$ by duality in $\pi_{0}$. We take the intersection of both sides with $\mathfrak{N}_{k}$ and take relative commutants in the type $I_{\infty}$ factor $\mathfrak{N}_{k}$ to get $\mathscr{R}\left(\mathscr{R}\left(\mathcal{O}_{1}\right)_{\mathfrak{N}_{k}}^{\prime},\left\{\left[\mathfrak{U}\left(\mathcal{O}^{x}\right) \cap \mathscr{R}\left(\mathcal{O}_{2}\right)\right]_{\pi_{0}}^{\prime} \cap \mathfrak{N}_{k}\right\}_{\mathfrak{N}_{k}}^{\prime}\right)=$ $\mathscr{R}(\mathcal{O})_{\pi_{0}}^{\prime} \cap \mathfrak{N}_{k}$. Since $\left[\mathfrak{U}\left(\mathcal{O}^{x}\right) \cap \mathscr{R}\left(\mathcal{O}_{2}\right)\right]_{\pi_{0}}^{\prime \prime} \subset \mathfrak{N}_{k}$ the second algebra on the left hand side in the last formula is equal to $\left[\mathfrak{U}\left(\mathcal{O}^{x}\right) \cap \mathscr{R}\left(\mathcal{O}_{2}\right)\right]_{\pi_{0}}^{\prime \prime}$. We then take $\mathcal{O}_{3} \gg \mathcal{O}_{2}$ such that $\mathscr{R}\left(\mathcal{O}_{1}\right)_{\mathfrak{N}_{k}}^{\prime}=\mathscr{R}\left(\mathcal{O}_{1}\right)_{\pi_{0}}^{\prime} \cap \mathfrak{N}_{k} \subset\left[\mathscr{R}\left(\mathcal{O}_{3}\right) \cap \mathfrak{U}\left(\mathcal{O}^{x}\right)\right]_{\pi_{0}}^{\prime \prime}$ and it follows that $\mathscr{R}(\mathcal{O})_{\pi_{0}}^{\prime} \cap \mathfrak{N}_{k}=$ $\mathscr{R}(\mathcal{O})^{c} \cap \mathfrak{N}_{k} \subset\left[\mathfrak{Q}\left(\mathcal{O}^{x}\right) \cap \mathscr{R}\left(\mathcal{O}_{3}\right)\right]_{\pi_{0}}^{\prime \prime}$. This construction can be carried out for infinitely many $\mathfrak{N}_{k}$ and the theorem is proved. 
Corollary Let $\pi_{\text {ren }}$ be an i.l.n.r. of the two dimensional CCR-algebra generated by a pure interacting vacuum state belonging to some $P(\Phi)_{2}$-model. Then duality holds for b.d.c.'s in $\pi_{r e n}$.

Remark. Clearly property $F$ is very strong, however, one would be quite content to have duality for i.l.n.r. which are Poincaré - or at least translation - covariant with the translations obeying spectrum condition. Therefore there is hope that for the physically interesting cases one can get away with less restrictive assumptions.

\section{Absence of Locally Generated Superselection Sectors ${ }^{3}$}

We begin with the following theorem whose consequences under more special conditions we shall explore later.

Theorem 2. Let $\mathfrak{A}$ have property $F$ and let $\omega$ be a pure, locally normal state on $\mathfrak{U}$ such that the corresponding GNS-vector $\Omega_{\omega}$ has the Reeh-Schlieder property ( $\omega$ is a "vacuum" state). Let $\rho$ be another pure, locally normal state such that for some b.d.c. $\mathcal{O}:\left.\rho\right|_{\mathscr{R}(\mathcal{O})^{c}}=\left.\omega\right|_{\mathscr{R}(\mathcal{O})^{c}}$. Then the representations $\pi_{\omega}$ and $\pi_{\rho}$ are unitarily equivalent. Furthermore, there is a vector $\Psi \in \mathscr{H}_{\rho}$ and a unitary $U$ in some local type I factor $\mathfrak{M}$ of $\mathfrak{U}$ such that $\rho_{\Psi}=\omega \circ A d U$.

Proof. (1) By the Reeh-Schlieder property for $\Omega_{\omega}$ together with equality of the states when restricted to $\mathscr{R}(\mathcal{O})^{c}$ we have for an arbitrary b.d.c. $\mathcal{O}_{1}$ with $\mathcal{O}_{1} \gg \mathcal{O}: \overline{\pi_{\rho}\left(\mathscr{R}\left(\mathcal{O}_{1}\right)^{c}\right) \Omega_{\rho}}=\overline{\pi_{\rho}\left(\mathscr{R}(\mathcal{O})^{c}\right) \Omega_{\rho}}$. Fix one such $\mathcal{O}_{1}$ and choose a type I factor $\mathfrak{M} \supset \mathscr{R}\left(\mathcal{O}_{1}\right)$. Then the projection $P$ onto $\overline{\pi_{\rho}\left(\mathfrak{M}^{c}\right) \Omega_{\rho}}$ is in $\mathfrak{M} \cap\left[\pi\left(\mathscr{R}(\mathcal{O})^{c}\right)\right]^{\prime}$ from which it easily follows that $P$ is infinite in $\mathfrak{M}$.

(2) Since $\mathfrak{M}$ is supposed to be countably decomposable $P$ is equivalent to the identity in $\mathfrak{M}([14]$, sec. III/8/6). Applying the corresponding partial isometry to $\Omega_{\rho}$ we get a vector $\Psi \in \mathscr{H}_{\rho}$ such that $\left.\rho\right|_{\mathfrak{M}^{c}}=\left.\rho_{\Psi}\right|_{\mathfrak{M}^{c}}$ and $\Psi$ is separating and cyclic for $\left[\pi_{\rho}\left(\mathfrak{M}^{c}\right)\right]^{\prime \prime}$. The map $\pi_{\rho}(A) \Psi \mapsto \pi_{\omega}(A) \Omega_{\omega}$ therefore extends to a unitary $W: \mathscr{H}_{\rho} \rightarrow \mathscr{H}_{\omega}$. From Lemma 1 of the preceding section we thus get $W \pi_{\rho}(\mathfrak{M}) W^{*}=$ $\pi_{\omega}(\mathfrak{M})$.

(3) From the argument in the proof of Theorem 1 it follows that the map $\pi_{\omega}(A) \mapsto \pi_{\rho}(A), A \in \mathfrak{M}$, also is an isomorphism between $\pi_{\omega}(\mathfrak{M})$ and $\pi_{\rho}(\mathfrak{M})$. Composing the two of them gives an automorphism of $\pi_{\rho}(\mathfrak{M})$ which is inner since $\mathfrak{M}$ is a type I factor ([15], 2.9.32).

Let $U \in \mathfrak{M}$ be the unitary which implements it. Composing this $U$ with $W$ we get a unitary $V$ such that $V \pi_{\rho}(A) V^{*}=\pi_{\omega}(A)$ for all $A$ which are either in $\mathfrak{M}$ or in $\mathfrak{M}^{c}$. But we can take $\mathfrak{M}$ to be the smallest member of an increasing sequence of type I factors whose union is dense in $\mathfrak{A}$. It is then immediately clear that $V \pi_{\rho}\left(\mathfrak{M}_{k}\right) V^{*}=\pi_{\omega}\left(\mathfrak{M}_{k}\right)$ for each $\mathfrak{M}_{k}$ in that sequence and thus: $V \pi_{\rho}(\mathfrak{U}) V^{*}=\pi_{\omega}(\mathfrak{A})$. The remaining assertion follows from the preceding arguments.

A drawback of this result is that we have to require $\left.\rho\right|_{\mathscr{R}(\mathcal{O})^{c}}=\left.\omega\right|_{\mathscr{R}(\mathcal{O})^{c}}$. It would clearly be preferable to use only the weaker assumption $\left.\rho\right|_{\mathfrak{q}\left(\mathscr{O}^{x}\right)}=\left.\omega\right|_{\mathfrak{a}\left(\mathscr{O}^{x}\right)}$. But 
even that can hardly be justified for $d=2$ where $\mathcal{O}^{x}$ is not connected. For $d \geqq 3$, however, we can do better since we have

Proposition 3. Let $\mathfrak{A}, \rho, \omega$ as in theorem 2 but suppose that merely $\left.\rho\right|_{\mathscr{R}(\tilde{\mathcal{O}})}=\left.\omega\right|_{\mathscr{R}(\tilde{\mathcal{O}})}$ for every b.d.c. $\widetilde{\mathcal{O}} \ll \mathcal{O}^{x}$. Then $\left.\rho\right|_{\mathfrak{A}\left(\Theta^{x}\right)}=\left.\omega\right|_{\mathfrak{I}\left(\Theta^{x}\right)}$.

Proof. For any b.d.c. $\widetilde{\mathcal{O}} \ll \mathcal{O}^{x}$ the map $\pi_{\rho}(A) \Omega_{\rho} \rightarrow \omega(A) \Omega_{\omega}, A \in \mathscr{R}(\widetilde{\mathcal{O}})$, defines an isometry $V_{\tilde{\mathfrak{O}}}: \overline{\pi_{\rho}(\mathscr{R}(\widetilde{\mathcal{O}})) \Omega_{\rho}} \rightarrow \overline{\pi_{\omega}(\mathscr{R}(\widetilde{\mathcal{O}})) \Omega_{\omega}}=\mathscr{H}_{\omega}$. From $\Omega_{\omega}$ having the ReehSchlieder property it then follows that $\frac{\omega}{\mathscr{R}\left(\widetilde{\mathcal{O}}_{1}\right) \Omega_{\rho}}=\overline{\mathscr{R}(\widetilde{\mathcal{O}}) \Omega_{\rho}}$ whenever $\widetilde{\mathcal{O}} \ll \widetilde{\mathcal{O}}_{1} \ll \mathcal{O}^{x}$.

Let now $\widetilde{\mathcal{O}}_{1}, \widetilde{\mathcal{O}}_{2} \subset \mathcal{O}^{x}$ and $\widetilde{\mathcal{O}}_{3} \subset \widetilde{\mathcal{O}}_{1} \cap \widetilde{\mathcal{O}}_{2}$, all b.d.c.'s, and let $P$ be the projection onto $\overline{\mathscr{R}\left(\widetilde{\mathcal{O}}_{1}\right) \Omega_{\rho}}$. So that by the previous argument $P$ is also the projection onto $\left.\overline{\mathscr{R}(\widetilde{\mathcal{O}}}{ }_{2}\right) \Omega_{\rho}$. We can find a complete orthonormal system $\left\{\Phi_{n}=A_{n} \Omega_{\rho}\right\}_{n \in \mathbb{N}}$ in $P \mathscr{H}_{\rho}$ where $\left\{A_{n}\right\} \subset \mathscr{R}\left(\widetilde{\mathcal{O}}_{3}\right)$. For $B \in \mathscr{R}\left(\widetilde{\mathcal{O}}_{1}\right), C \in \mathscr{R}\left(\widetilde{\mathcal{O}}_{2}\right)$ we then get:

$$
\begin{aligned}
\left(\Omega_{\rho}, \pi_{\rho}(B C) \Omega_{\rho}\right) & =\sum_{n}\left(\Omega_{\rho}, \pi_{\rho}\left(B A_{n}\right) \Omega_{\rho}\right)\left(\Omega_{\rho}, \pi_{\rho}\left(A_{n}^{*} C\right) \Omega_{\rho}\right) \\
& =\sum_{n}\left(\Omega_{\omega}, \pi_{\omega}\left(B A_{n}\right) \Omega_{\omega}\right)\left(\Omega_{\omega}, \pi_{\omega}\left(A_{n}^{*} C\right) \Omega_{\omega}\right)=\left(\Omega_{\omega}, \pi_{\omega}(A B) \Omega_{\omega}\right)
\end{aligned}
$$

since clearly $\left\{\pi_{\omega}\left(A_{n}\right) \Omega_{\omega}\right\}$ is a complete orthonormal system in $\mathscr{H}_{\omega}$. We can now proceed inductively to show that $\rho\left(\prod_{i=1}^{k} A_{i} B_{i}\right)=\omega\left(\prod_{i=1}^{k} A_{i} B_{i}\right)$ for all finite families $\left\{A_{i}\right\}_{1}^{k} \subset \mathscr{R}\left(\widetilde{\mathcal{O}}_{1}\right),\left\{B_{i}\right\}_{1}^{k} \subset \mathscr{R}\left(\widetilde{\mathcal{O}}_{2}\right)$.

By local normality we therefore get: $\left.\rho\right|_{\mathscr{R}\left(\mathscr{R}\left(\tilde{U}_{1}\right), \mathscr{R}\left(\tilde{\mathcal{O}}_{2}\right)\right)}=\left.\omega\right|_{\mathscr{R}\left(\mathscr{R}\left(\tilde{\mathcal{O}}_{1}\right), \mathscr{R}\left(\tilde{\mathcal{O}}_{2}\right)\right)}$. We can add further b.d.c.'s inductively and going over to the norm closure of $\bigcup_{\mathscr{U} \subset \mathscr{O}^{x}} \mathscr{R}(\widetilde{\mathcal{O}})$ we get the assertion.

Combining this with the results of Sect. 2 we get something resembling a quantum analogue of Derrick's theorem:

Theorem 4. Let $\mathfrak{A}$ have property $F$, let $\omega$ be a vacuum state on $\mathfrak{A}$ and assume $\pi_{\omega}$ has the properties of theorem 1. Let $\rho$ be another pure, locally normal state such that $\left.\rho\right|_{\mathscr{R}(\tilde{\mathcal{O}})}=\left.\omega\right|_{\mathscr{R}(\tilde{\hat{\theta}})}$ whenever $\tilde{\mathcal{O}}$ is a b.d.c. contained in $\mathcal{O}^{x}$ for some b.d.c. $\mathcal{O}$. Then $\pi_{\rho}$ and $\pi_{\omega}$ are unitarily equivalent.

Proof. By proposition $3 \rho$ and $\omega$ agree on $\mathfrak{A}\left(\mathcal{O}^{x}\right)$. By Theorem 1 duality holds in $\pi_{\rho}$, so there is a local type I factor $\mathfrak{M} \supset \mathscr{R}\left(\mathcal{O}_{1}\right), \mathcal{O}_{1} \gg \mathcal{O}$, and a $\Psi \in \mathscr{H} \rho$ which is separating and cyclic for $\pi_{\rho}\left(\mathfrak{M}^{c}\right)$ such that $\left.\rho_{\Psi}\right|_{\mathfrak{M}^{c}}=\left.\omega\right|_{\mathfrak{M}^{c}}$. One then proceeds as in the proof of Theorem 2 .

Let us now turn to examine the structure of the local system associated with the free complex scalar field. We won't go into any details which the interested reader can work out for himself either by consulting the explicit treatment in [16] or working from the abstract theory ([1], part I).

The complex field is a linear combination of two real fields: $\varphi_{c}(x)=\varphi_{1}(x)+$ $i \varphi_{2}(x)$. As local field algebras $\mathscr{F}(\mathcal{O})$ we may therefore take the tensor products of the corresponding local algebras of the real scalar field with themselves (we are not concerned here with the physical aspect of this choice). From standard results on tensor products of von Neumann algebras it then follows that this quasilocal system has all the nice properties discussed before. 
In order to get the observable algebras $\mathscr{R}_{c}(\mathcal{O})$ we have to average over the compact gauge group generated by the charge operator. The corresponding maps: $m_{\mathscr{O}}: \mathscr{F}(\mathcal{O}) \rightarrow \mathscr{R}_{c}(\mathcal{O})$ are normal C.E's for every b.d.c. $\mathcal{O}$. The vacuum representation of the quasilocal $C^{*}$-algebra $\mathfrak{I}_{c}$ one then gets by multiplying with the projection $P_{0}$ onto the charge zero subspace. Since $\mathfrak{U}_{c}$ is simple all its representations are isomorphisms. Let $\pi_{0}$ be the vacuum (charge zero) representation and $\pi_{\sigma}$ an irreducible representation with nonzero charge. These representations are inequivalent and duality holds in each of them since the conditions of thm. 4.1. in [1], part I, are fulfilled. Since furthermore the two representations are strongly locally equivalent and intertwined by a local automorphism of $\mathfrak{A}_{c}$ we can find a vector $\Psi_{\sigma} \in \mathscr{H}_{\sigma}$ such that for some b.d.c. $\mathcal{O} \Psi_{\sigma}$ is separating and cyclic for $\pi_{\sigma}\left(\mathfrak{U}\left(\mathcal{O}^{x}\right)\right)$ and the state on $\mathfrak{A}\left(\mathcal{O}^{x}\right)$ generated by $\Psi_{\sigma}$ coincides with the corresponding restriction of the vacuum state [1], part I, Sect. 6).

Let now $\mathcal{O}_{1}, \mathcal{O}_{2}$ be b.d.c.'s with $\mathcal{O}_{1} \ll \mathcal{O}_{2}$ and consider the von Neumann algebra $\mathscr{R}\left(\mathscr{F}\left(\mathcal{O}_{1}\right), \mathscr{F}\left(\mathcal{O}_{2}^{x}\right)^{\prime \prime}\right)$. There exists a normal product state $\rho$ on that algebra such that $\left.\rho\right|_{\mathscr{F}\left(\mathcal{O}_{1}\right)}=\left.\omega\right|_{\mathscr{F}\left(\mathcal{O}_{1}\right)}$ and $\left.\rho\right|_{\mathscr{F}\left(\mathcal{O}_{2}^{\times}\right)^{\prime \prime}}=\left.\omega\right|_{\mathscr{F}\left(\mathcal{O}_{2}^{x}\right)^{\prime \prime}}$. The restriction of $\rho$ to $\mathscr{R}\left(\mathscr{R}_{c}\left(\mathcal{O}_{1}\right)\right.$, $\left.\mathscr{R}_{c}\left(\mathcal{O}_{2}^{x}\right)^{\prime \prime}\right)$ is therefore a normal product state, too. This last algebra is isomorphic to its restriction to the vacuum sector and the vacuum vector $\Omega_{0}$ is separating for it, so $\rho$ is implemented by a vector $\Phi_{\rho} \in \mathscr{H}_{0}$. From duality in $\mathscr{H}_{0}$ it follows that $\mathfrak{U}_{c}$ has property $F$. What determines the existence or absence of nontrivial sectors is thus not property $F$ but Condition 2 of Theorem 1 which is not fulfilled by $\mathfrak{U}_{c}$. If it were equality of $\omega$ and $\left.\rho\right|_{\Psi \sigma}$ on $\mathfrak{A}_{c}\left(\mathcal{O}^{x}\right)$ would imply their equality on $\mathscr{R}_{c}(\mathcal{O})^{c}$ which we know is not the case.

But the following interesting consequence can be drawn for $\mathfrak{U}_{c}$ from the previous arguments. Let $\rho$ be a state on $\mathfrak{A}_{c}$ such as in Theorem 4 . Composing with the averaging map $m$ it follows that $\rho \circ m$ is such a state on $\mathscr{F}$. Since by Theorem 4 that last state is a Fock state we immediately get a uniqueness theorem for sectors with locally normal representations of $\mathfrak{U}_{c}$.

Acknowledgements. The author is very much indebted to L. Landau for the numerous discussions which helped the author a great deal to clarify his thoughts. Further thanks are due to J. Roberts for pointing out an error in an earlier version of this paper.

\section{References}

1. Doplicher, S., Haag, R., Roberts, J. E. : Commun. Math. Phys. 13, 1-23 (1969); Commun. Math. Phys. 15, 173-200 (1969)

2. Bisognano, J. J., Wichmann, E. H. : J. Math. Phys. 16, 985-1007 (1975)

3. Takesaki, M. : Pac. J. Math. 34, 807-816 (1970)

4. Buchholz, D. : Commun. Math. Phys. 36, 287-304 (1974)

5. Bonnard, J.-L., Streater, R. : Helv. Phys. Acta 49, 259-267 (1976)

6. Driessler, W., Fröhlich, J. : Ann. Inst. Henri Poincaré XXVII, 221-336 (1977)

7. Araki, H. : J. Math. Phys. 5, 1-13 (1964)

8. Goodman, R., Segal, I. : J. Math. Mech. 14, 629-638 (1965)

9. Rieffel, M. : Commun. Math. Phys. 39, 153-164 (1974)

10. Wilde, I. F. : Commun. Math. Phys. 24, 37-39 (1971)

11. Arveson, W. B. : Am. J. Math. 89, 578-642 (1967) 
12. Arveson, W. B. : Acta Math. 123, 141-224 (1969)

13. Borchers, H. J. : Commun. Math. Phys. 4, 315-323 (1967)

14. Dixmier, J. : Les algèbres d'operateurs dans l'espace Hilbertien 2nd. ed. Paris: Gauthier Villars, 1969

15. Sakai, S. : C*-and $W^{*}$-algebras. Berlin, Heidelberg, New York: Springer 1971

16. Wilde, I. F. : Aspects of algebraic quantum field theory. Lecture Notes, Instituto de Fisica Universidade de Sao Paulo, IFUSP/P-113

Communicated by K. Osterwalder

Received November 23, 1978 and in revised form March 10, 1979 